\title{
Effects of Non-Darcy Mixed Convection Over a Horizontal Cone With Different Convective Boundary Conditions Incorporating Gyrotactic Microorganisms On Dispersion
}

\author{
M. Ferdows ( $\nabla$ ferdows@du.ac.bd) \\ University of Dhaka \\ Bader Alshuraiaan \\ Kuwait University \\ Nayema Islam Nima \\ University of Dhaka
}

\section{Research Article}

Keywords: influence, transformations, microorganism, dispersion

Posted Date: November 30th, 2021

DOI: https://doi.org/10.21203/rs.3.rs-1080382/v1

License: (c) (i) This work is licensed under a Creative Commons Attribution 4.0 International License.

Read Full License 


\title{
Effects of non-Darcy mixed convection over a horizontal cone with different convective boundary conditions incorporating Gyrotactic microorganisms on dispersion
}

\author{
M. Ferdows ${ }^{1, *}$, Bader Alshuraiaan ${ }^{2}$ and Nayema Islam Nima $^{1}$ \\ ${ }^{1}$ Research Group of Fluid Flow Modeling and Simulation, Department of Applied Mathematics, \\ University of Dhaka, Dhaka-100, Bangladesh. \\ ${ }^{2}$ Department of Mechanical Engineering, Kuwait University, Safat 13060, Kuwait
}

\begin{abstract}
This paper discusses an investigation of the influence of dispersion impact on mixed convection flow over a horizontal cone within a non-Darcy porous medium subjected to convective boundary conditions. By imposing appropriate similarity transformations, the nonlinear partial differential equations governing flow, temperature, concentration, and microbe fields are reduced to a system of ordinary differential equations, which are then solved using the MATLAB BVP4C function. In a few circumstances, the research is brought to a strong conclusion by comparing the findings of the current study to previously published works. Mixed convection parameter $\lambda$, buoyancy parameters $N_{1}, N_{2}$, Lewis parameter Le, bioconvection lewis parameter Lb, Bioconvection peclet number Pe, Biot number $B_{i}$, Biot number of Mass transfer $B_{i, m}$ and also Biot number of motile microorganism transfer $B_{i, n}$ are all numerically calculated for various values of the dimensionless parameters of the problem. The results also reveal that, in the presence of dispersion effects, these parameters greatly influence the heat, mass, and motile microorganism transfer rates, as well as the corresponding velocity, temperature, concentration, and motile microorganism profiles.
\end{abstract}

\section{Introduction}

Convection in a porous fluid-saturated media from an axisymmetric bodies(such as a cone, cylinders, spheres) have attracted numerous researchers as a result of its numerous engineering applications and geophysics such as thermal insulation, geophysical flows, the cooling of electronic systems, ground water hydrology, chemical catalytic reactor, ceramic process, petroleum reservoirs, ground water pollution, filtration process etc. Complete reviews of convective heat transport in porous media have been written by Nield and Bejan [1], Pop and Ingham [2] and Bejan [3]. In the last few decades, a number of studies on mixed convective heat and mass transfer have been published, employing both Darcian and non-Darcian porous media.Srinivasachary and Reddy $[4,5]$ discovered natural and mixed convection for power law fluid in Darcy porous media, while Lai [6] used the Darcy model to observe mixed convection in porous media. Non-Darcian models are Darcy formulations that include inertial drag, vorticity diffusion, and combinations of these components, according to [7]. Various models for assessing 
non-Darcian flow in porous media, such as Brinkman-extended Darcy, Forchheimer-extended Darcy, and generalized stream models, have been published in the literature [7-11]. The non-Darcy model discussed in this study is a continuation of the classical Darcian formulation achieved by including a squared velocity factor to account for inertial effects in the momentum equation.

In all the above studies thermal dispersion effects are ignored. Many researchers [12-18] have looked at the influence of thermal dispersion on convective heat transfer through porous media in a variety of situations. Plumb [12] stated that when inertial effects are dominant, the thermal dispersion effects in a porous media become important. Effects of thermal dispersion on convection for non- Darcy porous media are studied by Amin [13] and Kairi [14]. And the effects of thermal dispersion for mixed convection flow with nanofluid through vertical surfaces are observed in refs. [16-18]. Heat and mass transfer analysis using a convective boundary condition is a significant and relevant consideration in the gas turbines, nuclear reactors, and heat exchangers industries. Heat is provided to the convecting fluid via a boundary surface with a finite heat capacity, which provides a convective heat transfer coefficient in this mechanism (namely, Biot number).Given the nature of these applications, Hady [19] studied non-Darcy natural convection with convective boundary condition containing microorganisms, mixed convection flow through vertical surfaces were studied in Refs. [20 - 23] in recent years and also With the presence of convective boundary conditions, Mahady [24] observed mixed convection boundary layer flow via a horizontal circular cylinder..

Now a day's researchers are paying more attention to the evolution of microorganisms in bioconvection. Bioconvection occurs when the macroscopic flow of fluid causes greater flexibility in swimming microorganisms. The self-driving mobile microorganisms desire to increase the volume of base fluid in the system by producing a bioconvective stream in one direction, according to Refs. [25-29]. Chemical or oxytactical properties, gyrotactic traits, and negative gravitational characteristics are used to classify motile microorganism. A bottom-heavy microbe with gyrotaxis is the most frequent. Kuznetsov [30] came up with the idea of putting motile microorganisms into nanofluid. The addition of motile microbes to the solution provides a number of benefits, including improved mass transfer, microscale mixing, and fluid stability. Refs. [31-38] provide important works on the use of bioconvection in thermobioconvection, microbial augmentation, bioMicrosystems, biofuels, and other bioengineering systems. Among them mixed convection containing gyrotactic microorganism flow with convective boundary conditions were observed in refs. [31-33] and also mixed convection phenomena with gyrotactic microorganism over cone were observed by Khan et al. [34], Saleem et al. [35]. Waqas [39] observed Magneto-Burgers Nanofluid Stratified Flow with Swimming Motile Microorganisms and also Hussian [40] studied MHD nanofluid flow with convective boundary conditions containing gyrotactic microorganism.

The objective of this article is to study free forced convection having motile gyrotactic microorganisms under the effects of dispersion past horizontal cone with convective boundary conditions which has not been studied yet. The main moto of this study are i) to examine mixed convection through heat mass and motile density ii) to analyze flow characteristics in the presence and absence of dispersion effects iii) to address multiple convective boundary conditions and also analyze the transfer rates of heat, mass, and motile microorganisms iv) to analyze the impact of 
different profile distributions for single mixed convection which encompasses the full mixed convection regime, from pure forced convection to pure free convection. v) to compare present results with previous published results in order to validate the accuracy of the present model.

\section{Model Formulation}

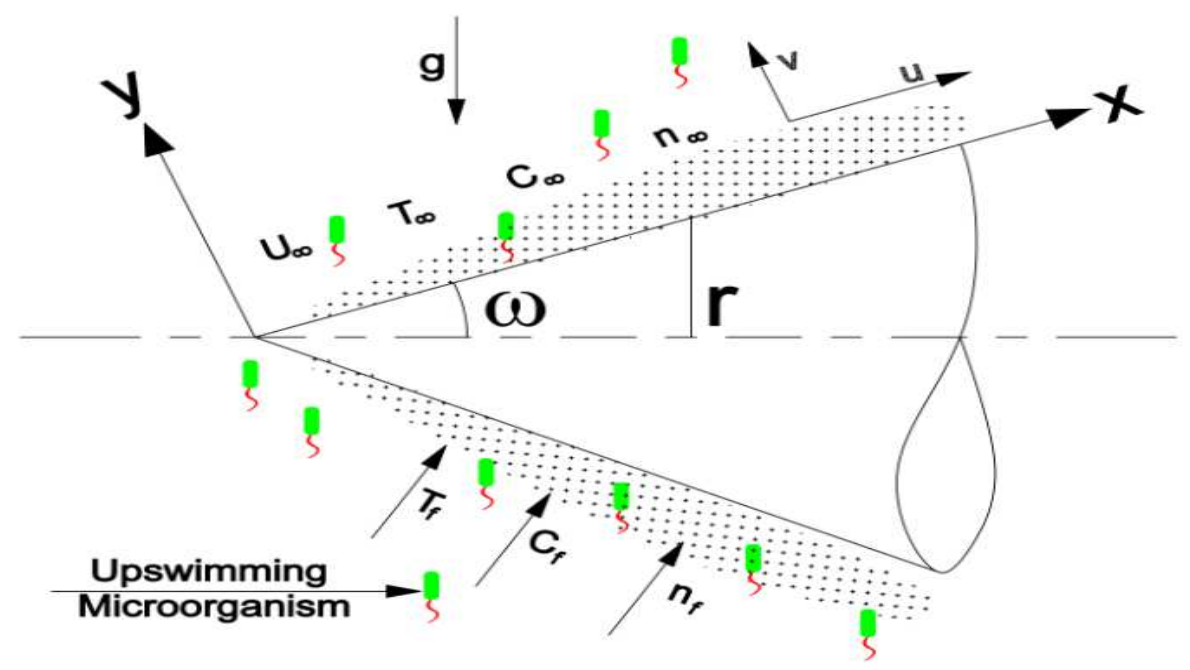

Fig1. Physical model

Consider the steady flow of mixed convection boundary layer past a semi-vertically angled horizontal cone immersed in a porous fluid-saturated media with ambient temperature $T_{\infty}$ and concentrations $C_{\infty}, n_{\infty}$. The coordinate $\mathrm{x}$ is measured from the cone's tip to the ray's end as well as the longitudinal coordinate $y$ is measured normal to it, see figure 1 . Convection from a warmed flowing fluid is expected to heat the cone's surface with gyrotactic microorganism at constant temperature $T_{f}$, constantconcentrations $C_{f}, n_{f}$ with variable heat, mass and motile microorganism coefficients $h_{f}(x), h_{m}(x)$ and $h_{n}(x)$ respectively. The diffusion-thermo and thermo-diffusion effects are also believed to be minimal at low concentrations of the diffusing species. According to refs. $[14,31,41]$ The conservation equations for the steady laminar boundary layer flow along a horizontal cone can be stated as the following using the non-Darcy model and Boussinesq approximation, as well as boundary layer approximations.

$\frac{\partial(r u)}{\partial x}+\frac{\partial(r v)}{\partial r}=0$ 


$$
\begin{aligned}
& \frac{\partial u}{\partial y}+\frac{\partial}{\partial y}\left(\rho \frac{b K^{*}}{\mu} u^{2}\right)=-\frac{\partial}{\partial x}\left(\frac{\rho g K \cos \beta}{\mu}\left[\beta_{T}\left(T-T_{\infty}\right)+\beta_{C}\left(C-C_{\infty}\right)+\beta_{n}\left(n-n_{\infty}\right)\right]\right) \\
& \left.u \frac{\partial T}{\partial x}+v \frac{\partial T}{\partial y}=\frac{\partial}{\partial y}\left(\alpha_{e} \frac{\partial T}{\partial y}\right)\right)(3) \\
& \left.u \frac{\partial C}{\partial x}+v \frac{\partial C}{\partial y}=\frac{\partial}{\partial y}\left(D_{c} \frac{\partial C}{\partial y}\right)\right) \\
& \left.u \frac{\partial n}{\partial x}+v \frac{\partial n}{\partial y}+\frac{b W_{c}}{C_{w}-C_{\infty}}\left(\frac{\partial}{\partial y}\left(n \frac{\partial C}{\partial y}\right)\right)=\frac{\partial}{\partial y}\left(D_{e} \frac{\partial n}{\partial y}\right)\right)
\end{aligned}
$$

With the boundary conditions are of the form:

$$
\begin{aligned}
& v=0,-k \frac{\partial T}{\partial y}=h_{f}(x)\left(T_{f}-T\right),-D_{m} \frac{\partial C}{\partial y}=h_{m}(x)\left(C_{f}-C\right), \\
& -D_{n} \frac{\partial n}{\partial y}=h_{n}(x)\left(n_{f}-n\right) \quad \text { at } \quad y=0(6) \\
& u \rightarrow u_{\infty}, T \rightarrow T_{\infty}, C \rightarrow C_{\infty}, n \rightarrow n_{\infty} \text { at } \quad y \rightarrow \infty
\end{aligned}
$$

Where $\mathrm{T}, \mathrm{C}, \mathrm{n}$ are temperature, concentration and volume fraction of motile microorganism. $\alpha_{e}, D_{c}, D_{e}$ are the effective thermal, solutal and microorganism diffusivities and these can be written as according to [14],

$$
\begin{aligned}
& \alpha_{e}=\alpha+a d u \\
& D_{c}=D_{m}+b d u \\
& D_{e}=D_{n}+c d u
\end{aligned}
$$

Where $\alpha, D_{m}, D_{n}$ are the constant thermal, molecular and microorganism diffusivities and $a, b, c$ are the coefficients of the thermal, solutal and microorganism dispersions sequentially. And the values of $a, b, c$ lie between $\frac{1}{7}$ and $\frac{1}{3}$.

Introducing the dimensionless quantities listed below

$$
\eta=\frac{y}{x} P e_{x}^{\frac{1}{2}}\left(1+\frac{R a_{x}^{\frac{1}{3}}}{P e_{x}^{\frac{1}{2}}}\right) \psi=\alpha r P e_{x}^{\frac{1}{2}}\left(1+\frac{R a_{x}^{\frac{1}{3}}}{P e_{x}^{\frac{1}{2}}}\right) f(\eta),
$$


$\theta(\eta)=\frac{T-T_{\infty}}{T_{f}-T_{\infty}}, \phi(\eta)=\frac{C-C_{\infty}}{C_{f}-C_{\infty}}, \chi(\eta)=\frac{n-n_{\infty}}{n_{f}-n_{\infty}}$

$h_{f}(x)=x^{-\frac{1}{2}} h_{f}, h_{m}(x)=x^{-\frac{1}{2}} h_{m}, h_{n}(x)=x^{-\frac{1}{2}} h_{n}$

Where $\psi$ is the stream function, as is customarily defined

$u=\frac{1}{r} \frac{\partial \psi}{\partial y}, v=-\frac{1}{r} \frac{\partial \psi}{\partial x}$

Transformed ordinary differential equations

$$
\begin{aligned}
& f^{\prime \prime}+2 \lambda^{2} \operatorname{Re} f f^{\prime \prime}+(1-\lambda)^{3}\left[-\frac{\eta \theta^{\prime}}{2}-\frac{\eta \theta^{\prime}}{6}(1-\lambda)+N_{1}\left(-\frac{\eta \phi^{\prime}}{2}-\frac{\eta \phi^{\prime}}{6}(1-\lambda)+N_{2}\left(-\frac{\eta \chi^{\prime}}{2}-\frac{\eta \chi^{\prime}}{6}(1-\lambda)\right]=0\right.\right. \\
& \theta^{\prime \prime}+\frac{1}{2} f \theta^{\prime}-\frac{f \theta^{\prime}}{6}(1-\lambda)+\lambda^{2} a P e_{d}\left(f^{\prime} \theta^{\prime \prime}+f^{\prime \prime} \theta^{\prime}\right)=0(10) \\
& \phi^{\prime \prime}+\frac{1}{2} L e f \phi^{\prime}-L e \frac{f \phi^{\prime}}{6}(1-\lambda)+L e \lambda^{2} b P e_{d}\left(f^{\prime} \phi^{\prime \prime}+f^{\prime \prime} \phi^{\prime}\right)=0(11) \\
& \chi^{\prime \prime}+\frac{1}{2} L b f \chi^{\prime}-L b \frac{f \chi^{\prime}}{6}(1-\lambda)+L b \lambda^{2} c P e_{d}\left(f^{\prime} \chi^{\prime \prime}+f^{\prime \prime} \chi^{\prime}\right)-P e\left[\phi^{\prime} \chi^{\prime}+(\chi+\omega) \phi^{\prime \prime}\right]=0(12)
\end{aligned}
$$

Boundary condition becomes

$$
\begin{aligned}
& f(0)=0, \theta^{\prime}(0)=-\lambda B_{i}(1-\theta(0)), \phi^{\prime}(0)=-\lambda B_{i, m}(1-\phi(0)), \chi^{\prime}(0)=-\lambda B_{i, n}(1-\chi(0)) a t \eta=0(13) \\
& f^{\prime}(\infty)=\lambda^{2}, \theta(\infty)=0, \phi(\infty)=0, \chi(\infty)=0 a t \eta \rightarrow \infty(14)
\end{aligned}
$$

WhereLocal Rayleigh number $R a_{x}=\frac{k g \beta_{T}\left(T_{f}-T_{\infty}\right) \cos \omega \cdot x}{v \alpha}$, Local peclet number $P e_{x}=\frac{u_{\infty} x}{\alpha}$, Mixed Convective term $\lambda=\frac{1}{R^{\frac{1}{3}}}$, Buoyancy Ratio parameter $N_{1}=\frac{\beta_{C}\left(C_{f}-C_{\infty}\right)}{\beta_{T}\left(T_{f}-T_{\infty}\right)}$, Buoyancy

$$
1+\frac{R a_{x}^{\frac{1}{3}}}{P e_{x}^{\frac{1}{2}}}
$$


Ratio parameter $N_{2}=\frac{\beta_{n}\left(n_{f}-n_{\infty}\right)}{\beta_{T}\left(T_{f}-T_{\infty}\right)}$, Lewis number $L e=\frac{\alpha}{D_{m}}$, Bioconvection Lewis number $L b=\frac{\alpha}{D_{n}}$, Bioconvection peclet number $P e=\frac{b W_{c}}{D_{n}}$, Microorganism concentration difference parameter $A=\frac{n_{\infty}}{n_{w}-n_{\infty}}$, Mixed convection parameter $\lambda=\frac{1}{1+\frac{R a_{x}^{\frac{1}{3}}}{P e_{x}^{\frac{1}{2}}}}$, Biot number $B_{i}=-\frac{h_{f} \alpha^{\frac{1}{2}}}{k u_{\infty}{ }^{\frac{1}{2}}}$

Biot number of mass transfer $B_{i, m}=\frac{h_{m} \alpha^{\frac{1}{2}}}{D_{m} u_{\infty}^{\frac{1}{2}}}$, Biot number of microorganism transfer $B_{i, n}=\frac{h_{n} \alpha^{\frac{1}{2}}}{D_{n} u_{\infty}^{\frac{1}{2}}}$, Inertia coefficient dependent Reynolds number $\operatorname{Re}=\frac{K^{*} b}{v} u_{\infty}$

The mixed convection parameter encompasses the full mixed convection regime, including pure free convection (when $\lambda=0\left(P e_{x}=0\right)$ ) to pure forced convection (when $\lambda=1\left(R a_{x}=0\right)$ ).

The local Nusselt number $N u_{x}$, Sherwood number $S h_{x}$ and local density number of the motile microorganisms $N n_{x}$ are expressed as

$N u_{x}=\frac{x q_{w}}{k\left(T_{f}-T_{\infty}\right)}, S h_{x}=\frac{x q_{m}}{D\left(C_{f}-C_{\infty}\right)}, N n_{x}=\frac{x q_{n}}{D_{n}\left(n_{f}-n_{\infty}\right)}$

Where the wall heat, wall mass, and wall motile microorganisms fluxes are specified as $q_{w}, q_{m}$, and $q_{n}$ respectively and the way they are defined as

$q_{w}=-k\left(\frac{\partial T}{\partial y}\right)_{y=0}, q_{m}=-D_{B}\left(\frac{\partial C}{\partial y}\right)_{y=0}, q_{n}=-D_{n}\left(\frac{\partial n}{\partial y}\right)_{y=0}$

The dimensionless forms of local nusselt number, local Sherwood number, and local motile microorganism are as follows,

$\lambda P e_{x}{ }^{\frac{-1}{2}} N u_{x}=-\theta^{\prime}(0), \lambda P e_{x}^{\frac{-1}{2}} S h_{x}=-\varphi^{\prime}(0), \lambda P e_{x}^{\frac{-1}{2}} N n_{x}=-\chi^{\prime}(0)$

\section{Numerical Method}


Simulation of the transformed Eqns. (9-12) within the confines of the boundary conditions(13)(14) are found for various values of the flow regulating parameters using the Matlab BVP4C numerical technique. The governing equations must be transformed into first order differential equations in the context of the bvp4c function stated above. At first Equations (9-12) can be rearranged in the following way

$$
\begin{aligned}
& f^{\prime \prime}=\frac{-(1-\lambda)^{3}\left(\frac{\lambda}{6}-\frac{2}{3}\right) \eta\left[\theta^{\prime}+N_{1} \phi^{\prime}+N_{2} \chi^{\prime}\right]}{\left(1+2 \operatorname{Re} f^{\prime}\right)} \\
& \theta^{\prime \prime}=-\left(\frac{\lambda}{6}+\frac{1}{3}\right) f \theta^{\prime}-\lambda^{2} a P e_{d}\left(f^{\prime} \theta^{\prime \prime}+f^{\prime \prime} \theta^{\prime}\right) \\
& \phi^{\prime \prime}=-\left(\frac{\lambda}{6}+\frac{1}{3}\right) L e f \phi^{\prime}-L e \lambda^{2} b P e_{d}\left(f^{\prime} \phi^{\prime \prime}+f^{\prime \prime} \phi^{\prime}\right) \\
& \chi^{\prime \prime}=-\left(\frac{\lambda}{6}+\frac{1}{3}\right) L b f \chi^{\prime}-L b \lambda^{2} c P e_{d}\left(f^{\prime} \chi^{\prime \prime}+f^{\prime \prime} \chi^{\prime}\right)+P e\left[\phi^{\prime} \chi^{\prime}+(\chi+A) \phi^{\prime \prime}\right]
\end{aligned}
$$

This equation must now be transformed into a first order differential equation. For this let $\eta=x$ and

$$
\begin{gathered}
y_{1}=f, \quad y_{2}=f^{\prime} \\
y_{3}=\theta, \quad y_{4}=\theta^{\prime}, \quad y_{5}=\phi, \\
y_{6}=\phi^{\prime}, \quad y_{7}=\chi, \quad y_{8}=\chi^{\prime}
\end{gathered}
$$


The first order differential equations are as follows:

$$
\begin{aligned}
& \frac{d y_{1}}{d x}=f^{\prime}=y_{2} \\
& \frac{d y_{2}}{d x}=f^{\prime \prime}=\frac{-(1-\lambda)^{3} \eta\left(\frac{\lambda}{6}-\frac{2}{3}\right)\left[y_{4}+N_{1} y_{6}+N_{2} y_{8}\right]}{\left(1+2 \lambda^{2} \operatorname{Re} y_{2}\right)} \\
& \frac{d y_{4}}{d x}=\theta^{\prime \prime}=\frac{-\left(\frac{\lambda}{6}+\frac{1}{3}\right) y_{1} y_{4}-\lambda^{2} a P e_{d} y_{4}\left(-\frac{-(1-\lambda)^{3} \eta\left(\frac{\lambda}{6}-\frac{2}{3}\right)\left(y_{4}+N_{1} y_{6}+N_{2} y_{8}\right)}{1+\lambda^{2} \operatorname{Re} y_{2}}\right)}{1+\lambda^{2} a P e_{d} y_{2}} \\
& \frac{d y_{6}}{d x}=\phi^{\prime \prime}=\frac{-L e\left(\frac{\lambda}{6}+\frac{1}{3}\right) y_{1} y_{6}-L e \lambda^{2} b P e_{d} y_{6}\left(-\frac{-(1-\lambda)^{3} \eta\left(\frac{\lambda}{6}-\frac{2}{3}\right)\left(y_{4}+N_{1} y_{6}+N_{2} y_{8}\right)}{1+\lambda^{2} \operatorname{Re} y_{2}}\right)}{1+\operatorname{Le}^{2} b P e_{d} y_{2}} \\
& -L b\left(\frac{\lambda}{6}+\frac{1}{3}\right) y_{1} y_{8}-L b \lambda^{2} c P e_{d} y_{8}\left(-\frac{(1-\lambda)^{3} \eta\left(\frac{\lambda}{6}-\frac{2}{3}\right)\left(y_{4}+N_{1} y_{6}+N_{2} y_{8}\right)}{1+\lambda^{2} \operatorname{Re} y_{2}}\right)+P e\left(y_{6} y_{8}+\right. \\
& \frac{d y_{8}}{d x}=\chi^{\prime \prime}=\frac{\left(y_{7}+A\right)\left(\frac{-L e\left(\frac{\lambda}{6}+\frac{1}{3}\right) y_{1} y_{6}-L e \lambda^{2} b P e_{d} y_{6}\left(-\frac{-(1-\lambda)^{3} \eta\left(\frac{\lambda}{6}-\frac{2}{3}\right)\left(y_{4}+N_{1} y_{6}+N_{2} y_{8}\right)}{1+\lambda^{2} \operatorname{Re} y_{2}}\right)}{1+L e \lambda^{2} b P e_{d} y_{2}}\right)}{1+L b \lambda^{2} c P e_{d} y_{2}}
\end{aligned}
$$

The boundary conditions become considering ya be the left boundary, $y b$ be the right boundary

$$
\begin{aligned}
& y a(1)=0, y b(2)-\lambda^{2}=0 \\
& y a(4)+\lambda B_{i}(1-y a(3))=0, y b(3)=0 \\
& y a(6)+\lambda B_{i, m}(1-y a(5))=0, y b(5)=0 \\
& y a(8)+\lambda B_{i, n}(1-y a(7))=0, y b(7)=0
\end{aligned}
$$

The MATLAB algorithm's accuracy and dependability have been proven in a number of recent research papers. Table 1 shows a clear comparison of the current findings with those of Reddy [22] for a few special circumstances, and there is a strong correlation. 


\begin{tabular}{|l|l|l|l|l|l|l|l|l|l|}
\hline \multicolumn{7}{|c|}{ Table 1: Comparison of $f^{\prime}(0),-\theta^{\prime}(0),-\phi^{\prime}(0)$ for $\lambda=1, \mathrm{Re}=0, P e_{d}=0, L b=0, P e=0, B_{i} \rightarrow \infty, B_{i, m} \rightarrow \infty$} \\
\hline \multicolumn{7}{|c|}{ When $L e=1$} & \multicolumn{2}{l|}{ When $L e=10$} \\
\hline$f^{\prime}(0)$ & $-\theta^{\prime}(0)$ & $-\phi^{\prime}(0)$ & $-\theta^{\prime}(0)$ & $-\phi^{\prime}(0)$ \\
\hline Reddy[22] & $\begin{array}{l}\text { Present } \\
\text { Result }\end{array}$ & Reddy[22] & $\begin{array}{l}\text { Present } \\
\text { Result }\end{array}$ & Reddy[22] & $\begin{array}{l}\text { Present } \\
\text { Result }\end{array}$ & Reddy[22] & $\begin{array}{l}\text { Present } \\
\text { Result }\end{array}$ & Reddy[22] & $\begin{array}{l}\text { Present } \\
\text { Result }\end{array}$ \\
\hline 1.0000 & 1.0000 & 0.5642 & 0.5644 & 0.5642 & 0.5644 & 0.5642 & 0.5644 & 1.7841 & 1.7841 \\
\hline
\end{tabular}

\section{Result discussion}

In Fig 2. The effect of mixed convection and buoyancy parameters on the velocity profile is demonstrated. With rising $\lambda$ values, the velocity profile increases in Fig.2 (a) which indicates velocity profile gradually increases for free to forced convection. Fig. 2(b-c) depicts that increase in buoyancy parameters $N_{1}, N_{2}$ resulting in an increase in the velocity profile. Because the buoyancy parameter is proportional to the buoyancy, the larger the buoyancy parameter, the greater the buoyancy. These higher levels of buoyancy operate as agents, increasing fluid velocity. And in every case it is observed velocity profile mostly increases for Darcy porous media comparing to non-Darcy case.

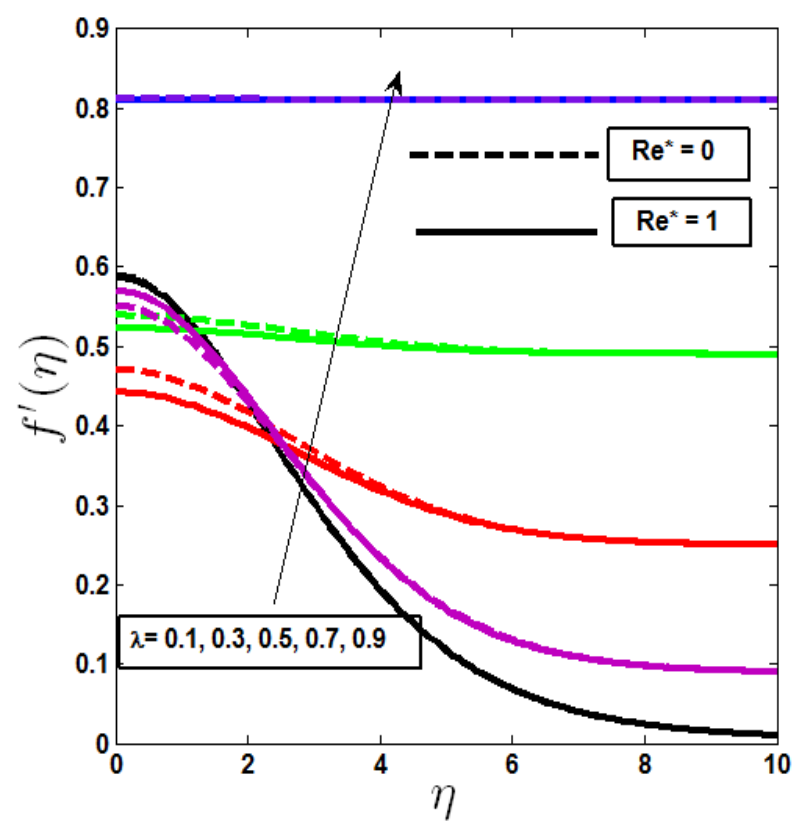

(a)

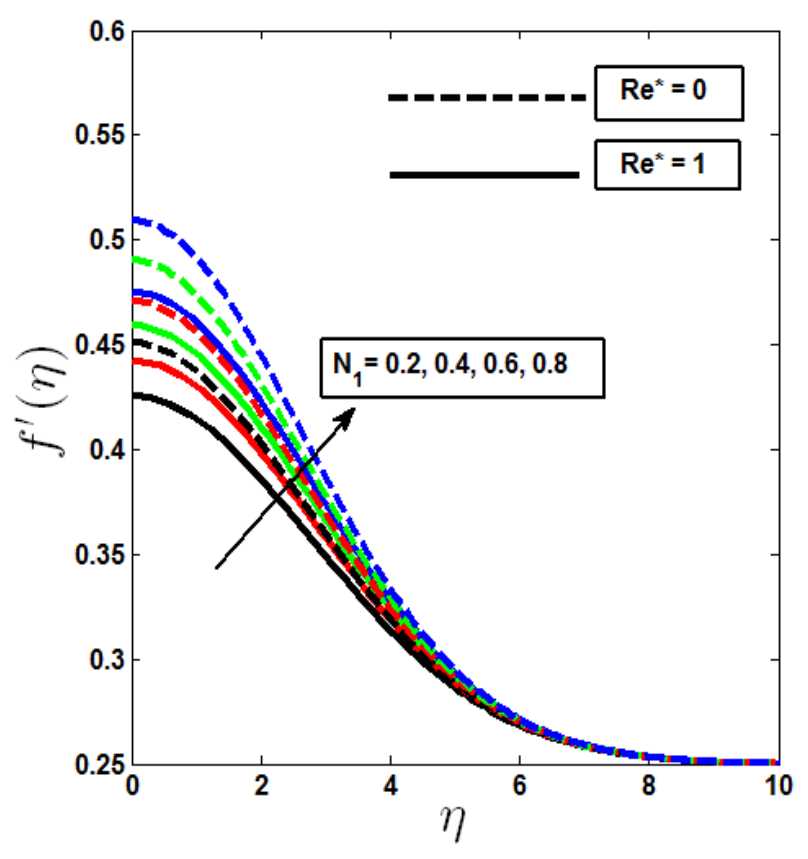

(b) 


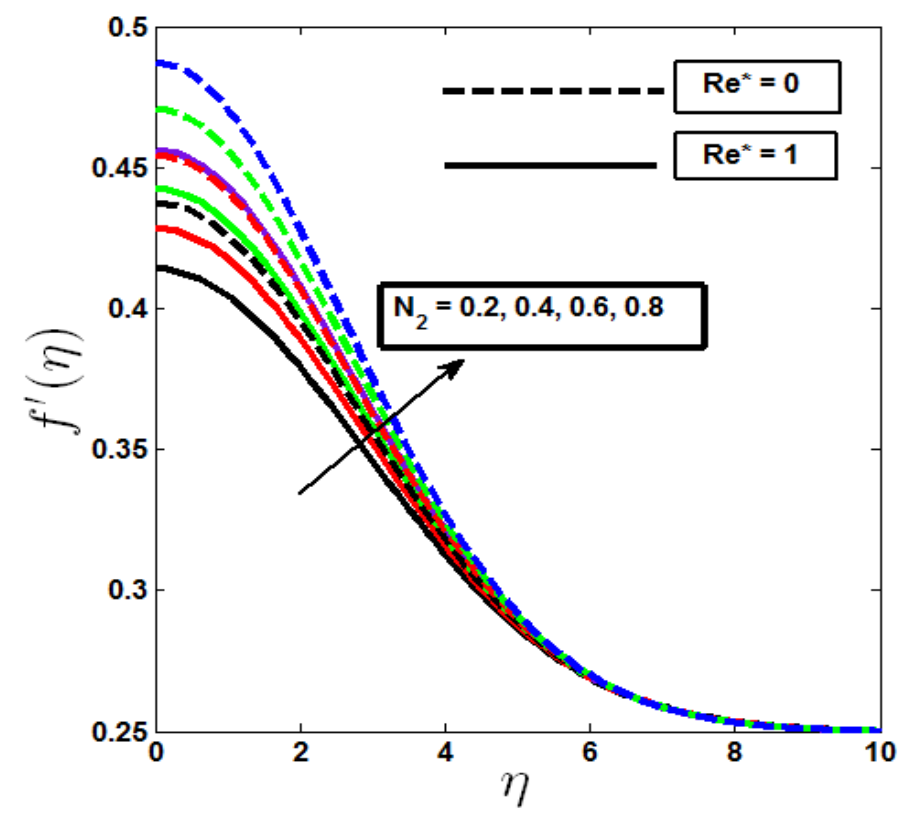

(c)

Fig 2. Velocity profile with the variation of a) Mixed convection parameter $\boldsymbol{\lambda}$ b) Buoyancy parameter $N_{1}$ c) Buoyancy parameter $N_{2}$ for Darcy and non-Darcy cases.

Effect of temperature profile with the growing values of mixed convection parameter $\boldsymbol{\lambda}$ and Biot number $B_{i}$ are observed in Fig 3. Fig 3(a) depicts temperature profile increases which mixed convection parameter $\lambda$ in the presence of dispersion effect but when dispersion effect is absent temperature profile increases from free to mixed convection regime, then again decreases to forced convection regime. In Fig 3(b) As the Biot number rises, the temperature profile rises with it. The Biot number helps to increase the temperature profiles of the fluid by increasing internal heat in solid surfaces. When there is a dispersion effect, the thickness of the boundary layer of the temperature profile increases. 

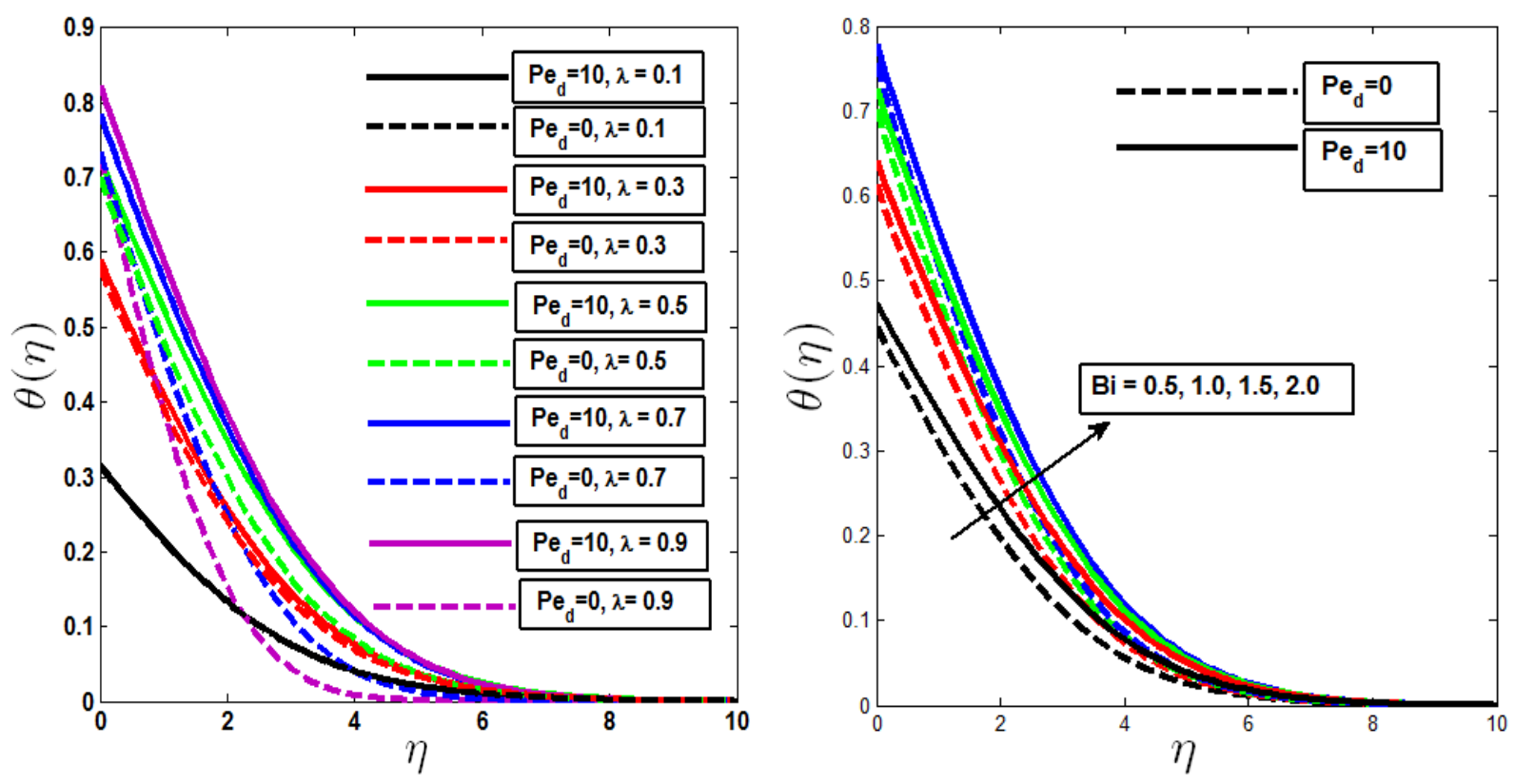

Fig 3. Temperature profile with the a) Variation of mixed convection parameter b) Biot number in the presence and absence of thermal dispersion effect.

The impact of the mixed convection parameter on concentration and microorganism profile is demonstrated in Figures 4(a) and 5(a). In both cases boundary layer thickness are increasing with $\lambda$ in the presence of dispersion effect. Without dispersion effect both profiles increases to pure free convection $(\boldsymbol{\lambda}=0)$ to pure mixed convection $(\boldsymbol{\lambda}=0.5)$ and then decreases to pure forced convection $(\lambda=1)$. Fig 4(b) and 5(b) depict that the concentration and microorganism profiles increase within the boundary layer when Biot number of mass transfer $B_{i, m}$ and Biot number of motile microorganism transfer $B_{i, n}$ increase from least to large value. Biot numbers are placed as a boundary condition in the enhanched wall boundary condition in eqn. (13). More heat and species of motile microorganisms are conveyed to the fluid as Biot Numbers rise, energizing the 
temperature, concentration, and microorganism profile boundary layer.
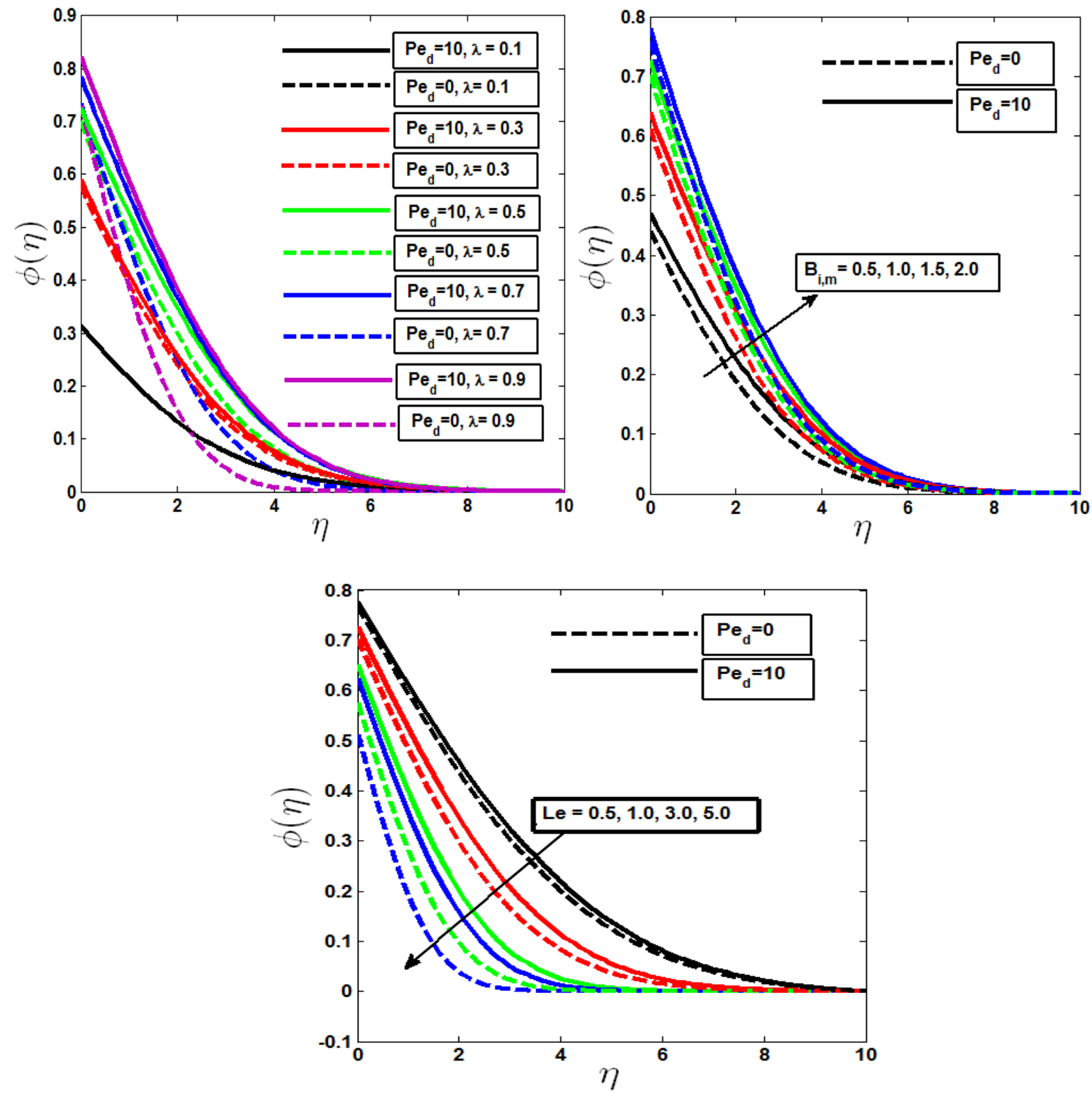

Fig 4. Concentration profile with the a) Variation of mixed convection parameter b) Biot number of mass transfer c) Lewis parameter Le in the presence and absence of solutal dispersion effect.

The ratio of thermal diffusivity to mass (Nano-particle) species diffusivity is known as the Lewis number. Le $=1$ denotes that the fluid's thermal diffusivity and species diffusivity are the same, as well as the thickness of both boundary layers. When Le is less than one, mass diffusivity is greater than thermal diffusivity, and vice versa when Le is greater. In Figure 4(c) The mass diffusivity of the concentration boundary layer decreases as the Lewis number decreases, lowering the penetration depth. And without the dispersion effect, the concentration profile deteriorates. Bioconvection Peclet number Pe and the bioconvection Lewis number Lb have a propensity to lower the motile microorganism density.Bioconvection peclet number Pe and The quantity of 
motile microbe thickness decreases as the Lewis number Lb of bioconvection increases fluid mobility which are shown in Fig 5(c) and 5(d). The behavior of Microorganism profile with and without dispersion effects are also shown in Fig 5.
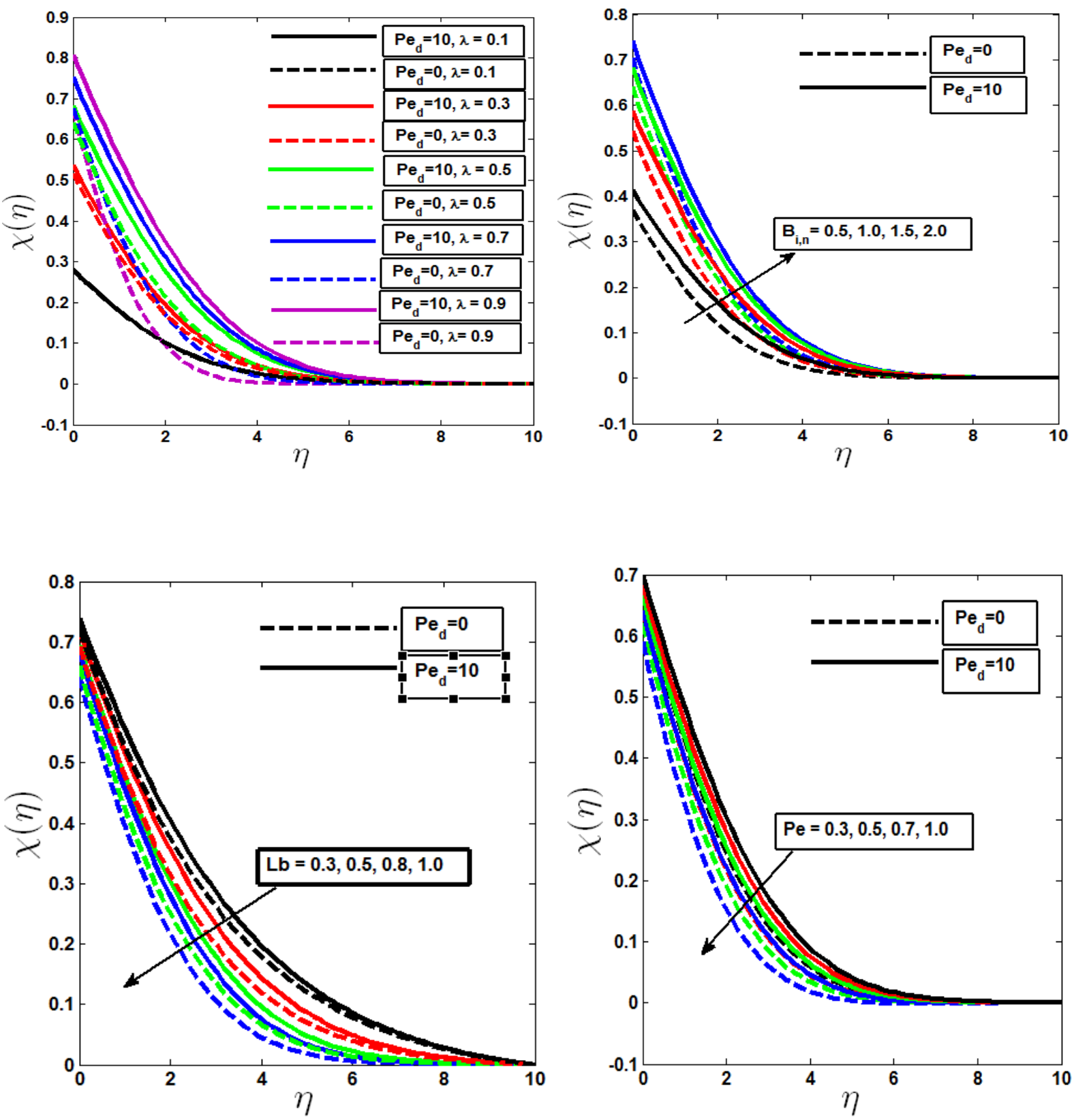

Fig 5. Microorganism profile with the a) Variation of mixed convection parameter b) Biot number of microorganism transfer c) Bioconvection Lewis parameter Le d) Bioconvection peclet number in the presence and absence of microorganism dispersion effect. 
Figure 6 shows the impacts of dispersion on heat, mass, and motile microbe transfer rates as the mixed convection parameter is varied. The rate of heat transmission increases as the dispersion parameter decreases. In Fig 6(a) it is observed that heat transfer rate is higher for the absence of dispersion effect. As we know $\boldsymbol{\lambda}$ is closer to 0 indicates free convection regime and closer to 1 indicates forced convection regime, in fig 6(a) it is noticeable that dispersion effect on heat transfer rate is negligible in free convection region. On the other hand effects are prominent in forced convection region. Similarly Fig 6(b) and 6(c) depict mass and motile microorganism transfer rate are increasing with the growing values of $\lambda$ when there is negligible dispersion effect. When $c=0.3$ and $P e_{d}=5$ mass and motile microorganism transfer rate increase from $\lambda=0$ to $\lambda=0.82$ and also whenc $=0.3$ and $P e_{d}=10$ mass and motile microorganism transfer rate increase from $\lambda=0$ to $\lambda=0.69$, after that in both cases mass and microorganism transfer rate gradually decrease. So decreasing phenomena is observed in forced convective region in the presence of dispersion effect.
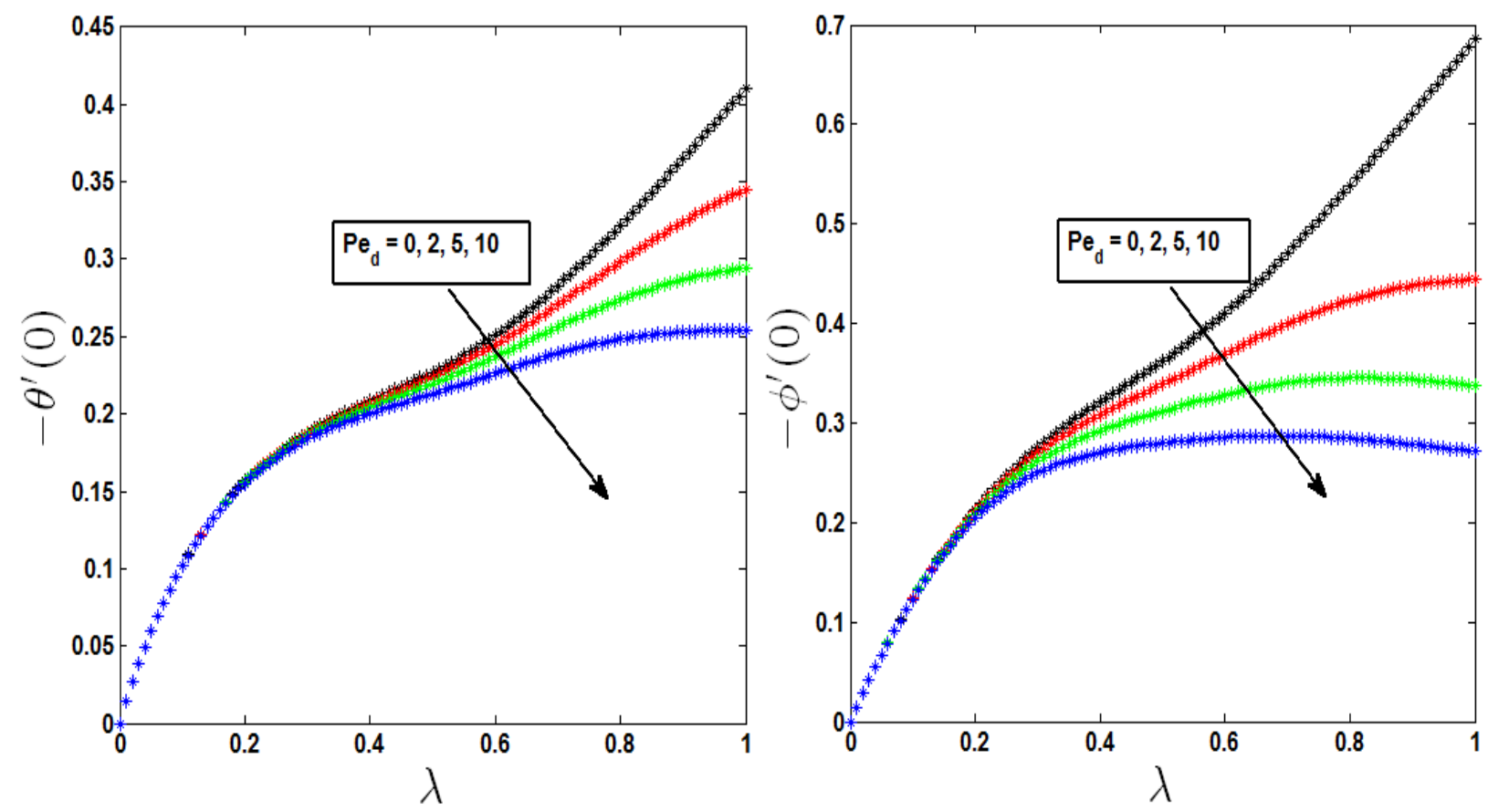


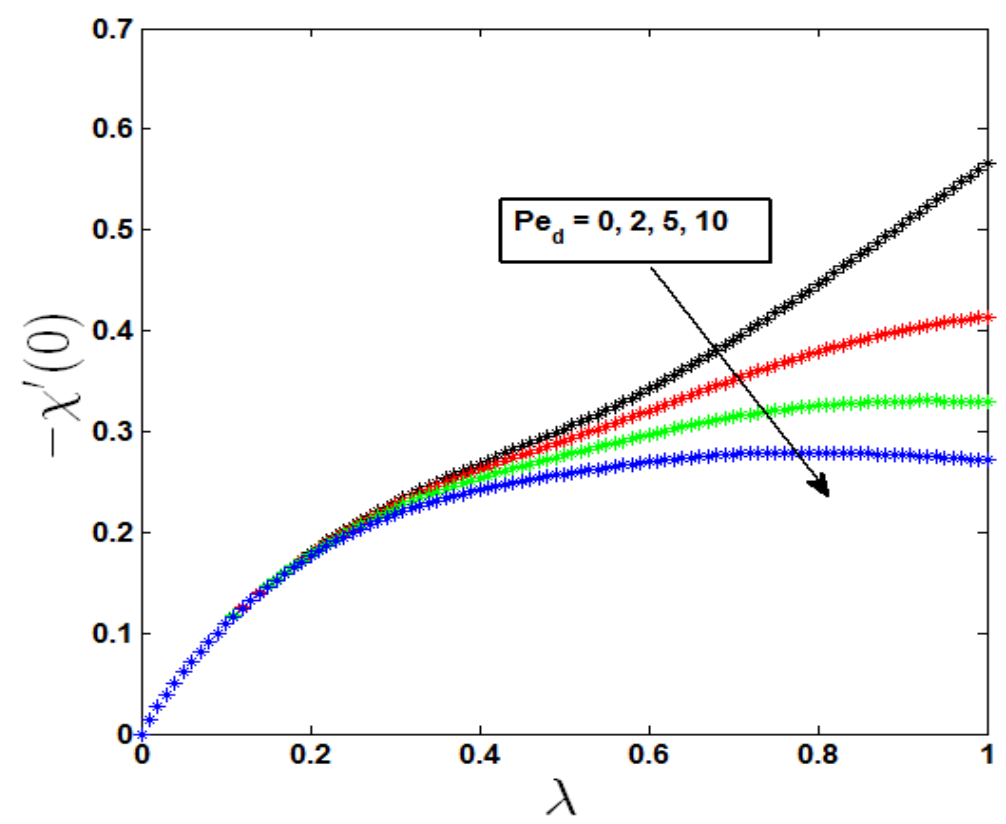

Fig 6. Dispersion effects on a) Heat transfer rate b) Mass transfer rate c) Motile Microorganism transfer rate in Free, Forced and Mixed convection regime.

For the growing effects of Biot numbers, heat, mass, and microbe transfer rates are observed in Fig 7 as the mixed convection parameter is varied. In every figure we can see heat, mass and motile microorganism transfer rates are increasing from $\lambda=0$ to $\lambda=1$ when dispersion effect is absent. But this increasing rate is not similar in all regions. In the mixed convection region it is seen in Fig 7 (a-c) that increasing phenomena is comparatively slower than free and forced convective region. On the otherhand when dispersion effects are present heat transfer rate decreases after when $B_{i}=1.5$ for $\lambda=0.98$ and $B_{i}=2$ for $\lambda=0.94$ which is closer to pure forced convection regime. And also mass transfer rate decreases after when $B_{i, m}=1$ for $\lambda=0.79 B_{i, m}=1.5$ for $\lambda=0.69$ and $B_{i, m}=2$ for $\lambda=0.62$. Similarly when $B_{i, n}=1$ for $\lambda=0.86 B_{i, n}=1.5$ for $\lambda=0.80$ and $B_{i, n}=2$ for $\lambda=0.74$, motile microorganism transfer rate drops. 

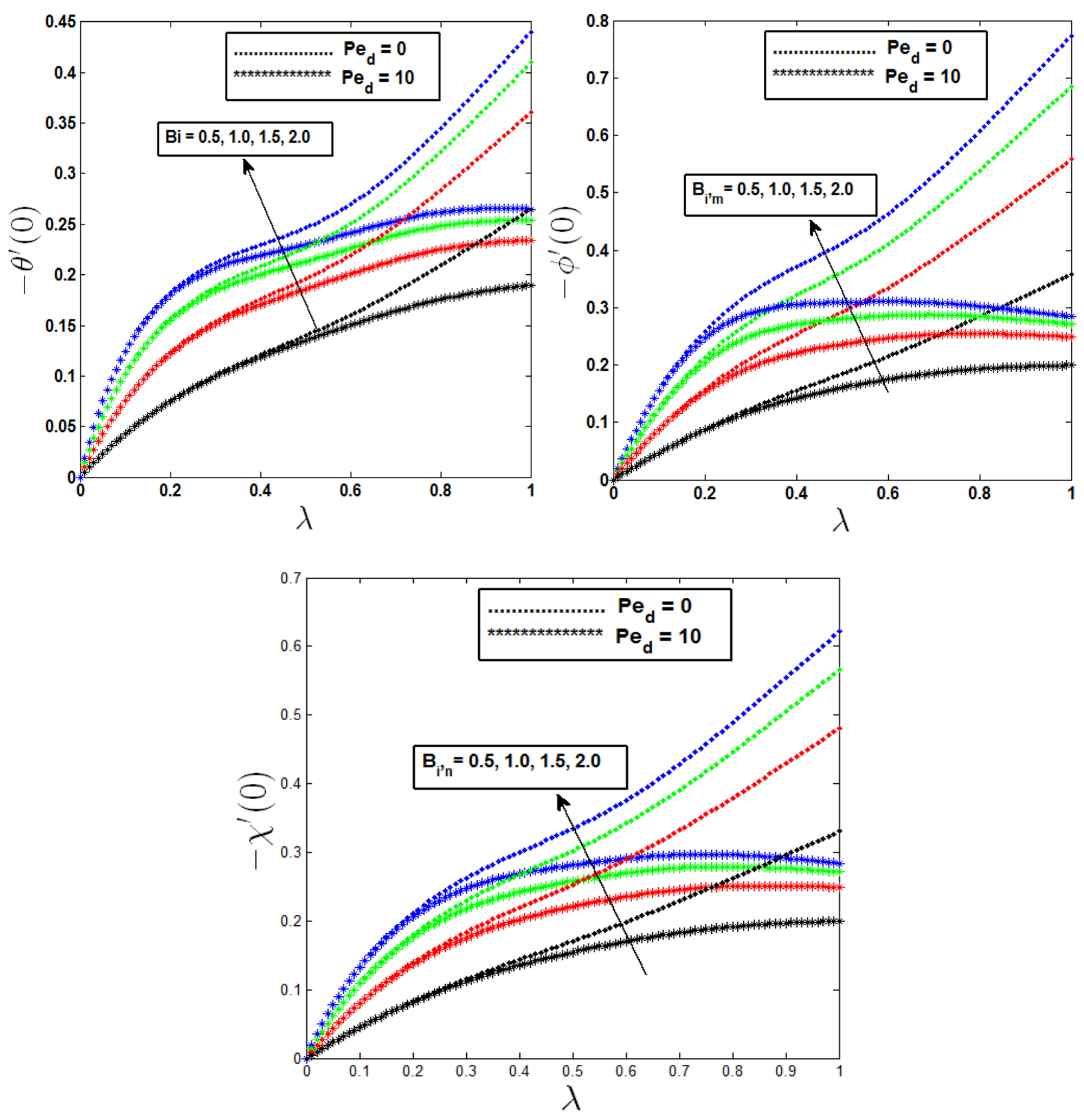

Fig 7. Effects of Biot numbers on a) Heat transfer rate b) Mass transfer rate c) Motile Microorganism transfer rate in Free, Forced and Mixed convection regime.

\section{Conclusion}

Theoretical and numerical studies are conducted on a new mathematical model for stable twodimensional mixed convection flow via a horizontal cone containing gyrotactic microorganisms with convective boundary conditions. The influences of dispersion effects on the rate of transport of heat, mass, and motile microorganisms along with velocity, temperature, concentration and 
microorganism profiles are observed. The key findings of this analysis can be summarized as follows:

- As the value of the mixed convection parameter $\lambda$ rises, the velocity profile and buoyancy parameters $N_{1}, N_{2}$ rise as well especially for Darcy porous media.

- Temperature, Concentration and Microorganism profiles are increasing with increasing values of $\boldsymbol{\lambda}$ when there is no dispersion effect. But in the presence of dispersion effect the profiles increase forfree $(\lambda=0)$ to pure mixed convection $(\boldsymbol{\lambda}=0.5)$, then decrease for those values of $\boldsymbol{\lambda}$ which indicate forced convection.

- Dispersion effect has great influence on the rate of heat, mass, and motile microbe transfer. For higher effects of dispersion the rate of heat, mass and motile microorganism transfer increase in a free-convection zone and drops in forced convection region.

- The biot number increases the rate of heat, mass and motile microorganism transfer from free to forced convection, with no influence on dispersion. The flow transfer rates decrease with the influence of the effect of dispersion.

\section{References}

[1] D. A. Nield and A. Bejan, Convection in PorousMedia, Springer,New York, NY, USA, 4th edition, 2013.

[2] I. Pop and D. B. Ingham, Convective Heat Transfer Mathematical and Computational Modeling of Viscous Fluids and Porous Media, Elsevier Science \& Technology Books, Pergaman, UK,2001.

[3] A. Bejan, Convection Heat Transfer, John Wiley, New York, NY,USA, 1994.

[4] Srinivasachary, D., \& Reddy, G.S. Double diffusive natural convection in power-law fluid saturated porous medium with Soret and Dufour Effects, Journal of the Brazilian Society of Mechanical Sciences and Engineering34(4), 525-530 (2012).

[5] Srinivasachary, D., \& Reddy, G.S. Mixed convection on a vertical plate in a power law fluid saturated porous medium with cross diffusion effects, Procedia Engineering 127, 591-597 (2015)

[6] Lai, F.C., Kulacki, F.A. \& Prasad, V. Mixed Convection in Saturated Porous Media. Convective Heat and Mass Transfer in Porous Media. NATO ASI Series (Series E: Applied Sciences196.(Springer, Dordrecht. 1991).

[7] Srinivasachary, D.\& Surender, O. Non- Darcy Mixed Convection in a Doubly Stratified porous medium with Soret Dufour effects. International Journal of Engineering Mathematics, Article ID 126218 (2014). 
[8] Ibrahim, F.S., Abdel-Gaid, S.M. and Subba Reddy Gorla, R., Non-Darcy mixed convection flow along a vertical plate embedded in a non-Newtonian fluid saturated porous medium with surface mass transfer. International Journal of Numerical Methods for Heat \& Fluid Flow 10(4), pp. 397-408 (2000).

[9] Kumari,P., Nigam,M., Kumar, S. et al. Magnetic field effect on non-Darcy mixed convection from a horizontal plate in a nanofluid -saturated porous medium. Journal of Porous Media 22(5): 599-610 (2019).

[10] Wang, Y\& Qin, G. Accurate numerical simulation for non-Darcy double-diffusive mixed convection in a double lid-driven porous cavity using SEM, Numerical Heat Transfer, Part A: Applications, 75:9, 598-615 (2019).

[11] Hayat, T., Haider, F. \& Alsaedi, A. Darcy-Forchheimer flow with nonlinear mixed convection. Appl. Math. Mech.-Engl. Ed. 41, 1685-1696 (2020).

[12] Plumb, O. The effect of thermal dispersion on heat transfer in packed bed boundary Layers, Proceedings of first ASME/JSME Thermal Engineering Joint Conference 2:17-21(1983).

[13] El-Amin, M. Double dispersion effects on natural convection heat and mass transfer in nonDarcy porous medium.Appl. Math. Comput. 156: 1-17 (2004).

[14] Kairi, R. R.VISCOSITY AND DISPERSION EFFECTS ON NATURAL CONVECTION FROM A VERTICAL CONE IN A NON-NEWTONIAN FLUID SATURATED POROUS MEDIUM. THERMAL SCIENCE 15(2) :S307-S316 (2012).

[15] Nasser, I. and H. Duwairi. "Thermal Dispersion Effects on Convection Heat Transfer in Porous Media with Viscous Dissipation.” International Journal of Heat and Technology 34: 207212 (2016).

[16] Khashi'ie, N.S., Md Arifin, N \& Pop, I. Non-Darcy mixed convection of hybrid nanofluid with thermal dispersion along a vertical plate embedded in a porous medium, International Communications in Heat and Mass Transfer, 118:104866 (2020).

[17] A. Aghbari, H. Ali Agha, D. Sadaoui, Soret-Dufour Effect on Mixed Convection Past a Vertical Plate in Non-Darcy Porous Medium Saturated With Buongiorno Nanofluid in the Presence of Thermal Dispersion, Journal of Mechanics 35(6):851-862 (2019).

[18] Meena OP. Mixed convection flow over a vertical cone with double dispersion and chemical reaction effects. Heat Transfer: 1-19 (2021).https://doi.org/10.1002/htj.22086.

[19]Hady., F.M., Mohamed. R.A., Mahdy, A., Abo Zaid \& Omima A. Non- Darcy Natural convection boundary layer flow over a vertical cone in porous media saturated with a nanofluid 
containing gyrotactic microorganisms with a convective boundary condition. Journal of nanofluids 5(5):765-773(2016).

[20] Rosali, H., Ishak, A., Nazar, R \& Pop, I. Mixed convection boundary layer flow past a vertical cone embedded in a porous medium subjected to a convective boundary condition. Propulsion and Power Research 5(2):118-122 (2016).

[21] Patil, P.M., Momoniat, E. \& Roy. S. Influence of convective boundary condition on double diffusive mixed convection from a permeable vertical surface. International Journal of Heat and Mass Transfer, 70:313-321(2014).

[22] RamReddy, C., Naveen, P. \& Srinivasacharya, D. Nonlinear Convective Flow of NonNewtonian Fluid over an Inclined Plate with Convective Surface Condition: A Darcy-Forchheimer Model. Int. J. Appl. Comput. Math 4, 51 (2018).

[23] Zainal,N.A. Nazar, R., Naganthran,K \& Pop,I. MHD mixed convection stagnation point flow of a hybrid nanofluid past a vertical flat plate with convective boundary condition. Chinese Journal of Physics 66: 630-644(2020).

[24] Mahat, R., Mahat, R., Rawi, N.A., Shafie, S and Kasim, A. R. M. Mixed Convection Boundary Layer Flow of Viscoelastic Nanofluid Past a Horizontal Circular Cylinder with Convective Boundary Condition. International Journal of Mechanical Engineering and Robotics Research 8(1): 87-91 (2019).

[25] Alloui, Z., Nguyen, T. H. \&Bilgen, E. Numerical Investigation of thermo-Bioconvection in a suspension of Gravitactic Microorganisms. Int. J. Heat Mass Transf. 56:1435-1441(2007).

[26] Avramenko, A. A. \& Kuznetsov, A. V. The onset of Bio-thermal convection in a suspension of Gyrotactic Microorganisms in a Fluid Layer with an Inclined Temperature Gradient. Int. J. Num. Methods Heat Fluid Flow. 20(1):111-129(2010).

[27] Khan, W.A., Uddin,M.J. \& Ismail, I.A. Free convection of non-Newtonian nano- fluids in Porous media with gyrotactic microorganisms. Transp Porous Med. 97:241-52(2013).

[28] Kuznetsov, A.V. The onset of Thermo-Bioconvection in a shallow Fluid Saturated porous Layer Heated from Below in a suspension of oxytactic microorganisms. Eur. J. Mech. B/ Fluids 25(2):223-233(2006).

[29] Kuznetsov, A.V. Bio-thermal convection induced by two different species of Microorganism. Intl. commun. Heat Mass Transf38(5):548-553(2011).

[30] Kuznetsov, A.V. The onset of nanofluid bioconvection in a suspension containing both nanoparticles and gyrotactic microorganisms. Int J Heat Mass Transf.37(10):1421-1425 (2010). 
[31]Latiff, N. A. A., Yahya, E., Ismail, A. I. M., Amirsom, A., and Basir, F., The effect of velocity slip and multiple convective boundary conditions in a Darcian porous media with microorganism past a vertical stretching/shrinking sheet. AIP Conference Proceedings1870(2017).

[32] Zaib, A.Rashidi, M.M \&Chamkha, Ali. J. Flow of nanofluid connatining gyrotactic microorganisms over static wedge in Darcy-Brinkman porous medium with convective boundary condition. Journal of Porous media 21(10) (2018).

[33] Rashad, A .M. and Hossain A. Nabwey. Gyrotactic mixed convection flow of a nanofluid past a circular cylinder with convective boundary condition. Journal of the Taiwan Instititute of chemical Engineerings99:9-17(2019).

[34] Khan, Waqar A., A.M. Rashad, M.M.M. Abdou and I. Tlili. Natural bioconvection flow of a nanofluid containing gyrotactic microorganisms about a truncated cone. European Journal of Mechanics - B/Fluids75: 133-142 (2019).

[35] Saleem S, HunzaRafiq,A. Al-Qahtani, Mohamed Abd El-Aziz, M.Y. Malik and I. L. Animasaun .Magneto Jeffrey Nanofluid Bioconvection over a Rotating Vertical Cone due to Gyrotactic Microorganisms.Mathematical Problems in Engineering 2019, Article ID: 3478037 :11 pages (2019).

[36] Sudnagar, P., Peri K. Kameswaran and B. Rushi Kumar. Gyrotactic Microorganism effects on mixed convective nanofluid flow past a vertical cylinder. Journal of thermal Science and Engineering Applications 11(4) : 11 pages (2019).

[37] Mahdy, A. and Hossam A. Nabwey. Microorganisms' time-mixed convection nanofluid flow by the stagnation domain of an impulsively rotating sphere due to Newtonian heating. Results in Physics 19:103347(2020).

[38] Mahdy, A. Unsteady Mixed Bioconvection Flow of Eyring-Powell Nanofluid with Motile Gyrotactic Microorganisms Past Stretching Surface. BioNanoSci. (2021).

[39] Waqas, H,.Manzoor,U., Shah, Z., Arif, M. \&Shutaywi, M. Magneto-Burgers Nanofluid Stratified Flow with Swimming Motile Microorganisms and Dual Variables Conductivity Configured by a Stretching Cylinder/Plate. Mathematical Problems in Engineering 2021, Article ID 8817435:16 pages (2021).

[40] Hussain, A. \& Malik, M.Y. MHD nanofluid flow over stretching cylinder with convectiveboundary conditions in the presence of gyrotactic swimming microorganism: A biomathematical model.International Communications in Heat and Mass Transfer 126:105425 (2021).

[41] Kumari, M \& Nath, G. Natural convection on a Horizontal Cone in a porous medium with non-Uniform wall temperature/concentration or Heat/Mass Flux and Suction/ Injection. Transp. Porous. Med.84:275-285(2010). 
